\title{
All-Dielectric Metasurface with Ultrahigh Color Filtering and Polarization-Independent/Dependent Characteristics
}

\section{Kunlin Chen}

Sun Yat-Sen University

\section{Rongpeng Fang}

Sun Yat-Sen University

\section{Yao Wen}

Sun Yat-Sen University

Yu-Sheng Lin ( $\square$ linyoush@mail.sysu.edu.cn )

School of Electronics and Information Technology, Sun Yat-Sen University https://orcid.org/00000002-2825-0955

\section{Nano Express}

Keywords: dielectric nanostructure, color filter (CF), tunability, filtering and polarization micro-LED,

Posted Date: June 22nd, 2021

DOl: https://doi.org/10.21203/rs.3.rs-622397/v1

License: (9) This work is licensed under a Creative Commons Attribution 4.0 International License. Read Full License 


\section{Abstract}

All-dielectric nanostructure-based color filter (CF) has attracted huge interest in many fields due to its excellent optical properties. We present four metasurface-based CFs with different shapes on quartz substrates to generate pure blue, green and red colors. CFs show maximum reflection intensities of $99.6 \%, 99.7 \%$, and $95.1 \%$ and the corresponding spectra bandwidths are $13 \mathrm{~nm}, 16 \mathrm{~nm}$, and $14 \mathrm{~nm}$ for blue, green and red colors, respectively. The proposed metasurface-based CFs possess high color saturations and ultra-narrow bandwidths. In addition, metasurface-based CFs with different shapes show the tunability for color switching characteristics. These CFs designs will potentially benefit reflective display technologies, and their ultrahigh color filtering and polarization-independent/dependent characteristics are particularly useful for high resolution color printing, micro-LED, polarizer-switches, tunable color switches, and so on.

\section{Introduction}

Metasurface-based color filters (CFs) have drawn widely attentions with the improvement of nanofabrication technologies due to the better optical properties than those using pigments and organic colors [1-3]. There are many metasurface-based CFs are presented to generate blue, green, and red colors filtering characteristics including all-dielectric, metallic, insulator/metal/insulator, and metal-insulatormetal nanostructures, etc. The physical mechanisms are surface plasmon resonances generated between the incident light and the metallic nanostructures, or Mie resonances generated within all-dielectric nanostructures [4-7]. On account of the development of the nanofabrication technologies, there are various shapes of nanostructures, such as nanoparticles, nanocubes, nanogratings, nanowires, nanoholes, nanodisks, and nanopillars are extensively studied to achieve the CFs with excellent optical performances [8-13]. Among these CFs, the metasurface-based CFs possess excellent color filtering characteristics, which have been demonstrated in widespread applications, such as macroscopic color hologram, polarizer, high-resolution color printing, anticounterfeiting, color switches, information encoding, display, image sensor, and so on [14-20].

Since the metallic nanoparticles are pioneers employed in the nanostructure-based $\mathrm{CFs}$, the plasmonic gold (Au), silver (Ag), and aluminum (Al) materials are usually used to create various CFs with different nanostructures [21, 22]. However, Au and Ag materials are replaced by Al material because of the interband transition of Au in the short visible wavelength below $550 \mathrm{~nm}$ and the lower stability of $\mathrm{Ag}$ easily being oxidized and sulfureted [22-25]. Instead, Al not only shows more robust for the fabrication process and cost-effective in promising large-scale economical manufacturing, but also has broad resonance range and strong resonant intensity due to the interband transition located at the infrared (IR) wavelength of about $800 \mathrm{~nm}$ and near-UV wavelength range [24, 25]. Although Al has such good properties, but the significant intrinsic ohmic losses are inevitable occurred for all-metal plasmonic nanostructures due to the material absorption of Al [22, 24-25]. Therefore, the researches on dielectric materials have been studied to overcome the defects of using superimposed metal-dielectric or all-metal nanostructures. [25-27]. To compare with the superimposed metal-dielectric materials, all-dielectric 
materials are easier to be manufactured. They are low cost without metal materials and less fabrication steps. Particularly, all-dielectric CFs can generate bright and pure colors due to the low absorption loss. Among these dielectric materials, silicon ( $\mathrm{Si}$ ) based CFs are widely investigated since it is low-cost, reliable, mature fabrication process, and facile to integrate into the optoelectronics applications $[4,24]$. However, Si has strong absorption in the visible wavelength range smaller than $450 \mathrm{~nm}$. Therefore, $\mathrm{SiO}_{2}$, $\mathrm{TiO}_{2}, \mathrm{Si}_{3} \mathrm{~N}_{4}$, and polymer-based materials become the alternative materials for all-dielectric nanostructures [27].

In this study, all dielectric metasurface using $\mathrm{TiO}_{2}$ nanodisks is presented to perform $\mathrm{CFs} \mathrm{TiO}_{2}$-nanodisks CFs can be generated vivid blue, green, and red colors by changing the $\mathrm{TiO}_{2}$ geometrical parameters according to the theory of Mie resonances. There are four types of $\mathrm{TiO}_{2}$-nanodisks $\mathrm{CFs}$ are proposed, which are tailored with nanodisk, nanoring, split-disk, and fan configurations denoted as CF-1, CF-2, CF-3, and CF-4, respectively. The corresponding electromagnetic characteristics are investigated and discussed by using three-dimensional finite difference time domain (FDTD) based simulations.

\section{Design And Method}

Figure 1(a-d) show the schematic drawings of CF-1, CF-2, CF-3, and CF-4, respectively. All geometrical parameters of CFs are also defined in Fig. 1. They are the diameter $(R)$, height $(h)$, and period $(P)$ of nanodisk as shown in Fig. 1(a), diameter $(R)$, height $(h)$, period $(P)$, and inner diameter $(r)$ of nanoring as shown in Fig. 1(b), diameter $(R)$, height $(h)$, period $(P)$, and gap $(d)$ of split-disk as shown in Fig. 1(c), diameter $(R)$, height $(h)$, period $(P)$, included angle $(a)$ of fan as shown in Fig. 1(d), respectively. The propagation direction of incident light is set along negative $z$-axis direction in the numerical simulations. The periodic boundary conditions are adopted in the $\mathrm{x}$ - and $\mathrm{y}$-axis directions, and perfectly matched layer $(\mathrm{PML})$ boundaries conditions are assumed in the $z$-direction.

\section{Results And Discussions}

Figure 2(a) and (b) show the reflection and transmission spectra of RGB colors of CF-1 device. The resonances of RGB colors are $457 \mathrm{~nm}(B), 560 \mathrm{~nm}(\mathrm{G})$, and $704 \mathrm{~nm}(\mathrm{R})$ under the geometrical conditions of $R=220 \mathrm{~nm}(\mathrm{~B}), 250 \mathrm{~nm}(\mathrm{G}), 350 \mathrm{~nm}(\mathrm{R}), h=110 \mathrm{~nm}(\mathrm{~B}), 150 \mathrm{~nm}(\mathrm{G}), 150 \mathrm{~nm}(\mathrm{R})$, and $P=310 \mathrm{~nm}(\mathrm{~B})$, $380 \mathrm{~nm}(\mathrm{G}), 480 \mathrm{~nm}(\mathrm{R})$, respectively. The reflection intensities of RGB colors are all approximate $100 \%$ and the corresponding full width at half maximum (FWHM) values are less than $16 \mathrm{~nm}$. All resonances, geometrical parameters, FWHM values, transmission and reflection intensities of CF-1 are summarized in Table I. These results show that CF-1 exhibits ultrahigh color filtering characteristics in the visible spectrum.

Table I. Detailed geometrical parameters and spectra information of RGB colors filtering characteristics of CF-1. 


\begin{tabular}{lllllll}
$\begin{array}{l}\text { Resonance } \\
(\mathrm{nm})\end{array}$ & $\begin{array}{l}R \\
(\mathrm{~nm})\end{array}$ & $\begin{array}{l}h \\
(\mathrm{~nm})\end{array}$ & $\begin{array}{l}P \\
(\mathrm{~nm})\end{array}$ & $\begin{array}{l}\text { Reflection } \\
\text { Intensity }\end{array}$ & $\begin{array}{l}\text { Transmission } \\
\text { Intensity }\end{array}$ & \\
\hline 457 & 220 & 110 & 310 & 0.996 & 0.0063 & 13 \\
\hline 560 & 250 & 150 & 380 & 0.997 & 0.0039 & 16 \\
\hline 704 & 350 & 150 & 480 & 0.951 & 0.0475 & 14
\end{tabular}

Figure 3 shows the electric (E) field distributions on $x y$ - and xz-planes of RGB colors filtering characteristics in TE and TM modes for CF-1 device. For xy-plane, the E-field energies are mainly concentrated in the gap of two nanodisks along y-direction in TE mode and $x$-direction in TM mode. It indicates that this resonant behavior is a dipole resonance. By increasing the resonant wavelength from blue to red spectrum, E-field distribution appears gradually in the nanodisk. These results are corresponded with the reflection and transmission spectra as shown in Fig. 2. From the cross-sectional views (xz-plane) of E-field distributions, E-field energies of green and red spectra are mainly concentrated in the nanodisk for TE mode and the interface of nanodisk and substrate for TM mode. In particular, the resonant intensities of TE and TM modes are very strong for blue spectrum, which are distributed on top and bottom sides of nanodisk for TE mode and gap between nanodisks for TM mode.

The resonant intensity of $\mathrm{TiO} 2$ metasurface can be effectively enhanced by adjusting and increasing the aggregation degree of $\mathrm{TiO} 2$ scatterers [27]. Therefore, the structural color can be generated by using periodic TiO2 nanodisks. Moreover, the coupling effect of Mie resonances with photonic crystal radiation can help to enhance the reflection intensity and decrease the FWHM value. These effects are useful to design ultrahigh color filtering devices. In view of the theories of Mie resonances and the results in Fig. 2 and Fig. 3, the reason of minor resonances appearing in green and red spectra is owing to the increment of period, the gap between two adjacent TiO2 nanodisks also becomes larger. The numbers of Mie scatters and scattering intensity will be reduced. The enhanced efficiency of resonances is also be attenuated. Therefore, there will produce minor resonances at shorter wavelength.

To investigate the influence of geometrical parameters of CF-1, Fig. 4(a) and (b) show the reflection spectra of CF- 1 with different $P$ and $h$ values for green color filtering characteristic, respectively. In Fig, 4(a), by increasing $P$ value from $350 \mathrm{~nm}$ to $400 \mathrm{~nm}$, the resonances are red-shifted and the resonant intensities are decreased gradually. The minor resonances appear at the wavelengths of $512 \mathrm{~nm}$ ad 525 $\mathrm{nm}$ under the conditions of $P=350 \mathrm{~nm}$ and $360 \mathrm{~nm}$. They will disappear when $P$ becomes larger. The reason is when other physical parameters determined, the amount of Mie scatters generated between CF1 is fixed. CF-1 will be more compact under the condition of smaller $P$ value, and the Mie resonant intensity will be stronger. Therefore, it generates minor resonances at lower wavelengths. In Fig, 4(b), by increasing $h$ value from $140 \mathrm{~nm}$ to $240 \mathrm{~nm}$, the resonances are red-shifted and the resonant intensities 
are kept as stable. The minor resonances gradually appear at the wavelength of $560 \mathrm{~nm}$ when the $h$ value is bigger than $160 \mathrm{~nm}$. It is because of all geometrical parameters are constant except the $h$ parameter of CF-1. The Mie scatter mode is existed and the scatters intensities are enhanced by the increasing $h$ value, which plays an important role in the Mie resonances along with the generation of another scatter mode. These results prove that the Mie resonances of CF- 1 are determined by the geometrical parameters of nanodisk.

Figure 5(a-c) are the contour maps of reflection spectra of CF-1 with different incident angles for blue, green, and red colors filtering characteristics. The resonant intensities are obviously influenced by the incident angle, which decrease gradually by increasing the incident angle. The resonant intensities are higher than 0.55 for blue spectrum that the incident angle is larger than $60^{\circ}$. For green and red spectra, the resonant intensity higher than 0.55 is only $30^{\circ}$ and will be attenuated at larger incident angles. They possess stronger incident angle-dependent characteristic. The physical mechanism is that $h$ value for blue spectrum is smaller than that for green and red spectra, the influence of incident angle is less. Figure $5(d-f)$ are the contour maps of reflection spectra of CF-1 with different polarization angles for blue, green, and red colors filtering characteristics. All resonant intensities are stable by changing polarization angles. It indicates that the CF-1 device is polarization-independence due to the symmetrical structure of CF-1. This highly polarization-independent characteristic is one of the key performance indicators of modern display technologies.

Figure 6 shows the reflection intensities of CF-2 with different $r$ values for RGB colors filtering characteristics. Due to the nanoring structure of CF-2 is symmetric and similar to that of CF-1, CF-2 also possesses polarization-independent characteristic. For RGB spectra, when $r$ value increases to a certain amount, the reflection intensities start to decline gradually. It is because the increment of $r$ value will reduce the resonant area and then attenuate the effect of E-field coupling accordingly. The inserted E-field images indicate the reflection intensities of blue and green colors filtering characteristics are stronger than that of red color filtering characteristic due to the smaller physical parameters to constitute more compact $\mathrm{TiO}_{2}$ nanoring. Therefore, the coupling effect of E-field is stronger and then resulted in the higher reflection intensities.

Figure 7 shows the reflection intensities of CF-3 with different $d$ values for RGB colors filtering characteristics in TE and TM modes. By increasing $d$ values, all reflection intensities decrease firstly and then increase finally. The $d$ value increases until to be tangent to the adjacent split-disk, there will generate new Mie scatters that as same as the original ones and then have the same resonant intensity. It can be found that the same trends for both TE and TM modes, but the resonant intensity recovers to the highest intensity in TM mode is relatively smaller than that in TE mode. The reason is the E-field coupling effect in TM mode is smaller than that in TE mode. However, the amount of Mie scatters is almost the same for both modes, which will generate the same resonances.

Figure 8 illustrates the reflection spectra of CF-4 with different $a$ values for RGB colors filtering characteristics in TE (Fig. 8(a-c)) and TM (Fig. 8(d-f)) modes. By increasing $a$ value, the empty area of CF- 
4 increases. The E-field energy confined within the fan shape decreases gradually and then resulted in a reflection intensity decrease for blue, green, and red colors filtering characteristics, respectively. All reflection intensities in TE mode (Fig. 8(a-c)) are stronger than those in TM mode (Fig. 8(d-f)) due to the Efield distributions of TE resonance is larger than those of TM resonance as the inserted E-field images shown in Fig. 8. Particularly, when $a=30^{\circ}$, there is a significant difference in the reflection intensity between TE mode and TM mode. Such results mean that CF-4 has certain polarization-dependent characteristics. In addition, the resonances are almost stable not changed by increasing $a$ value in TE and TM modes.

\section{Conclusion}

In conclusion, four types of $\mathrm{TiO}_{2}$-nanodisk based metasurface $\mathrm{CFs}$ are proposed to perform $\mathrm{RGB}$ colors ultrahigh-efficiency filtering characteristics. The optical properties of CFs could be designed to possess polarization-dependent/independent characteristics. The reflection intensities of CF-1 are 0.996, 0.997, and 0.951 for blue, green, and red colors filtering characteristics and the corresponding FWHM values are $13 \mathrm{~nm}, 16 \mathrm{~nm}$, and $14 \mathrm{~nm}$, respectively. CF-2 exhibits the same excellent color filtering and polarizationindependent characteristics as CF-1. CF-3 and CF-4 have great potentials for polarization switching application due to their polarization-dependent characteristics. Such excellent performances of CFs demonstrate that they are applicable to be used in high resolution color printing, micro-LED, polarizerswitches, tunable color switches, and so on.

\section{Declarations}

\section{Availability of Data and Material}

All data generated or analyzed during this study are included in this published article.

\section{Competing Interests}

The authors declare that they have no competing interests.

\section{Funding}

Natural Science Foundation of Basic and Applied Foundation of Guangdong Province (2021A1515012217), National Key Research and Development Program of China (2019YFA0705004), and National Natural Science Foundation of China (11690031).

\section{Authors' Contributions}


$\mathrm{KC}, \mathrm{RF}$, andYW carried out the related experiments and data analysis. KC and RF drafted the manuscript. YSL supervised the experiments and the revising of the manuscript. YSL also provided suggestions and guidance for the experiments and data analysis. All authors read and approved the final manuscript.

\section{Acknowledgements}

The authors acknowledge the State Key Laboratory of Optoelectronic Materials and Technologies of Sun Yat-Sen University for the use of simulation codes.

\section{Authors information}

\section{Affiliations: School of Electronics and Information Technology, Sun Yat-Sen University, Guangzhou, 510006, China.}

- Kunlin Chen†

- Rongpeng Fang†

- Yao Wen

- Yu-Sheng Lin*

\section{References}

1. K. Wen, X. Jiang, J. He, G. Li, and J. Yang,Color-Adjustable Devices Based on the SurfacePlasmons Effect, Appl. Sci. 10, 1960 (2020).

2. J. Su, L. Wang, J. Yang, L. Kong, X. Mo, and J. Lv, High selectivity plasmonic color filters based on tapered annular aperturearrays, Opt. Commun. 475, 126206 (2020)

3. X. L. Hu, L. B. Sun, B. Shi, M. Ye, Y. Xu, L. S. Wang, J. Zhao, X. L. Li, Y. Q. Wu, S. M. Yang, R. Z. Tai, H.J. Fecht, J. Z. Jiang, and D. X. Zhang, Influence of film thickness and nanograting period on colorfilter behaviorsof plasmonic metal Ag films, J. Appl. Phys. 115, 113104 (2014)

4. Z.-J. Zhao, M. Lee, H. Kang, S. Hwang, S. Jeon, N. Park, S.-H. Park, and J.-H. Jeong, Eight Inch WaferScale Flexible Polarization-Dependent Color Filters with $\mathrm{Ag}-\mathrm{TiO}_{2}$ Composite Nanowires, ACS Appl. Mater. Interfaces 10, 9188-9196 (2018).

5. V. R. Shrestha, S.-S. Lee, E.-S. Kim, and D.-Y. Choi, Aluminum Plasmonics Based Highly Transmissive Polarization-Independent Subtractive Color Filters Exploiting a Nanopatch Array, Nano Lett. 14, 6672-6678 (2014).

6. A. Hosseini, and Y. Massoud, A low-loss metal-insulator-metalplasmonic bragg reflector, Opt. Express 14(23), 11318-11322 (2006).

7. J. Proust, F. Bedu, B. Gallas, I. Ozerov, and N. Bonod,All-Dielectric Colored Metasurfaces with Silicon Mie Resonators, ACS Nano 10, 7761-7767 (2016). 
8. H.-S. Lee, Y.-T. Yoon, S.-S. Lee, S.-H. Kim, and K.-D. Lee, Color filter based on a subwavelength patterned metal grating, Opt. Express 15(23), 15457- 15463 (2007).

9. Y. F. Yu, A. Y. Zhu, R. P.- Domínguez, Y. H. Fu, B. Luk'yanchuk, and A. I. Kuznetsov, High-transmission dielectric metasurface with $2 \pi$ phase control at visible wavelengths, Laser Photonics Rev. 9(4), 412418 (2015).

10. J. J. Wang, W. Zhang, X. Deng, J. Deng, F. Liu, P. Sciortino, and L. Chen, High-performance nanowiregrid polarizers, Opt. Express 30(2), 195- 197 (2005).

11. J.-H. Yang, V. E. Babicheva, M.-W. Yu, T.-C. Lu, T.-R. Lin, and K.-P. Chen,Structural Colors Enabled by Lattice Resonance on Silicon Nitride Metasurfaces, ACS Nano 14, 5678-5685 (2020)

12. M. K. Hedayati, M. Elbahri,Review of Metasurface Plasmonic Structural Color, Plasmonics 12, 14631479 (2017).

13. F. Gildas, and Y. Dan, Review of nanostructure color filters, J. Nanophoton. 13(2), 020901 (2019).

14. H.-S. Koo, M. Chen, P.-C. Pan, LCD-based color filter films fabricated by a pigment-based colorant photoresist inks and printing technology, Thin Solid Films 515, 896-901 (2006).

15. S. Yokogawa, S. P. Burgos, and H. A. Atwater, Plasmonic Color Filters for CMOS Image Sensor Applications, Nano Lett. 12, 4349-4354 (2012).

16. T. Xu, Y.-K. Wu, X. Luo, and L. J. Guo,Plasmonic nanoresonators for high-resolution colour filtering and spectral imaging, Nat. Commun. 1, 1-5 (2010).

17. M. C. Gather, A. Köhnen, A. Falcou, H. Becker, and K. Meerholz, Solution-Processed Full-Color Polymer Organic Light-Emitting Diode Displays Fabricated by Direct Photolithography, Adv. Funct. Mater. 17, 191-200 (2007).

18. Y.-S. Lin, J. Dai, Z. Zeng, and B.-R. Yang, Metasurface Color Filters Using Aluminumand Lithium Niobate Configurations, Nanoscale Res. Lett. 15, 77 (2020)

19. J. Wang, Q. Fan, S. Zhang, Z. Zhang, H. Zhang, Y. Liang, X. Cao, and T. Xu, Ultra-thin plasmonic color filters incorporating free-standing resonantmembrane waveguides with high transmission efficiency, Appl. Phys. Lett. 110, 031110 (2017)

20. X. Zhang, Y.-S. Lin, and B.-R. Yang, Tunable color switch using split-ring metamaterial, Opt. Laser Technol. 131, 106461 (2020)

21. Eunso Shin, Seokhyeon Hong, Young Jin Lee and Soon-Hong Kwon, Achieving full color with a strong Mie resonance using a silicon U-shape nanostructure metasurface, J. Opt. 21(5), 055105 (2019).

22. B. Yang, W. Liu, Z. Li, H. Cheng, S. Chen, and J. Tian,Polarization-Sensitive Structural Colors with Hueand-Saturation Tuning Based on All-Dielectric Nanopixels, Adv. Optical Mater. 6, 1701009 (2018)

23. V. R. Shrestha, S.-S. Lee, E.-S. Kim, and D.-Y. Choi, Polarization-tuned Dynamic ColorFilters Incorporating a Dielectric-loadedAluminum Nanowire Array, Sci. Rep. 5, 12450 (2015)

24. V. Vashistha, G. Vaidya, R. S. Hegde, A. E Serebryannikov, N. Bonod, and M. Krawczyk, All-Dielectric Metasurfaces Based on Cross-Shaped Resonators for Color Pixels with Extended Gamut, ACS Photonics 4, 1076-1082 (2017). 
25. V. Vashistha, G. Vaidya, P. Gruszecki, A. E. Serebryannikov, and M. Krawczyk, Polarization tunable alldielectric color filters based on cross-shapedSi nanoantennas, Sci. Rep. 7, 8092 (2017)

26. M. J. Uddin, and R. Magnusson, Highly efficient color filter array using resonant $\mathrm{Si}_{3} \mathrm{~N}_{4}$ gratings, Opt. Express 21(10), 12495-12506, (2013)

27. S. Sun, Z. Zhou, C. Zhang, Y. Gao, Z. Duan, S. Xiao, and Q. Song,All-Dielectric Full-Color Printing with $\mathrm{TiO}_{2}$ Metasurfaces, ACS Nano 11, 4445-4452 (2017).

\section{Figures}

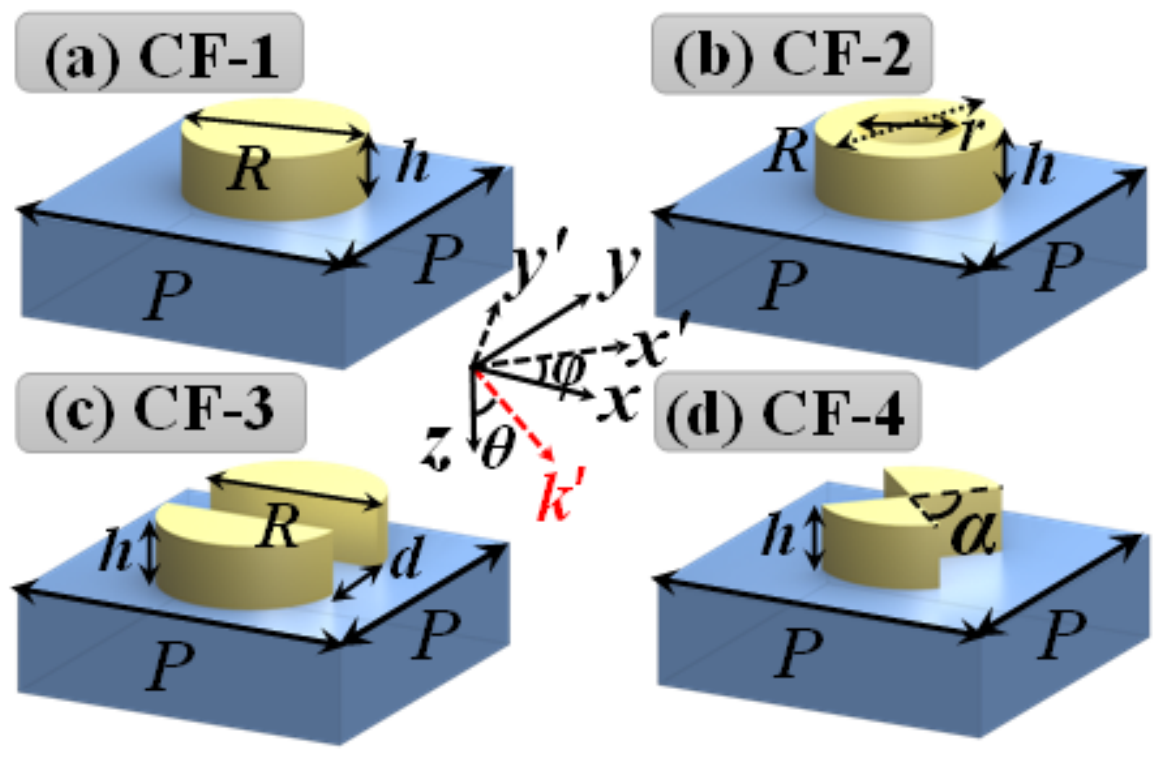

Figure 1

Schematic drawings of TiO2-nanodisks CFs on SiO2 substrates. They are (a) CF-1, (b) CF-2, (c) CF-3, and (d) CF-4, respectively. 

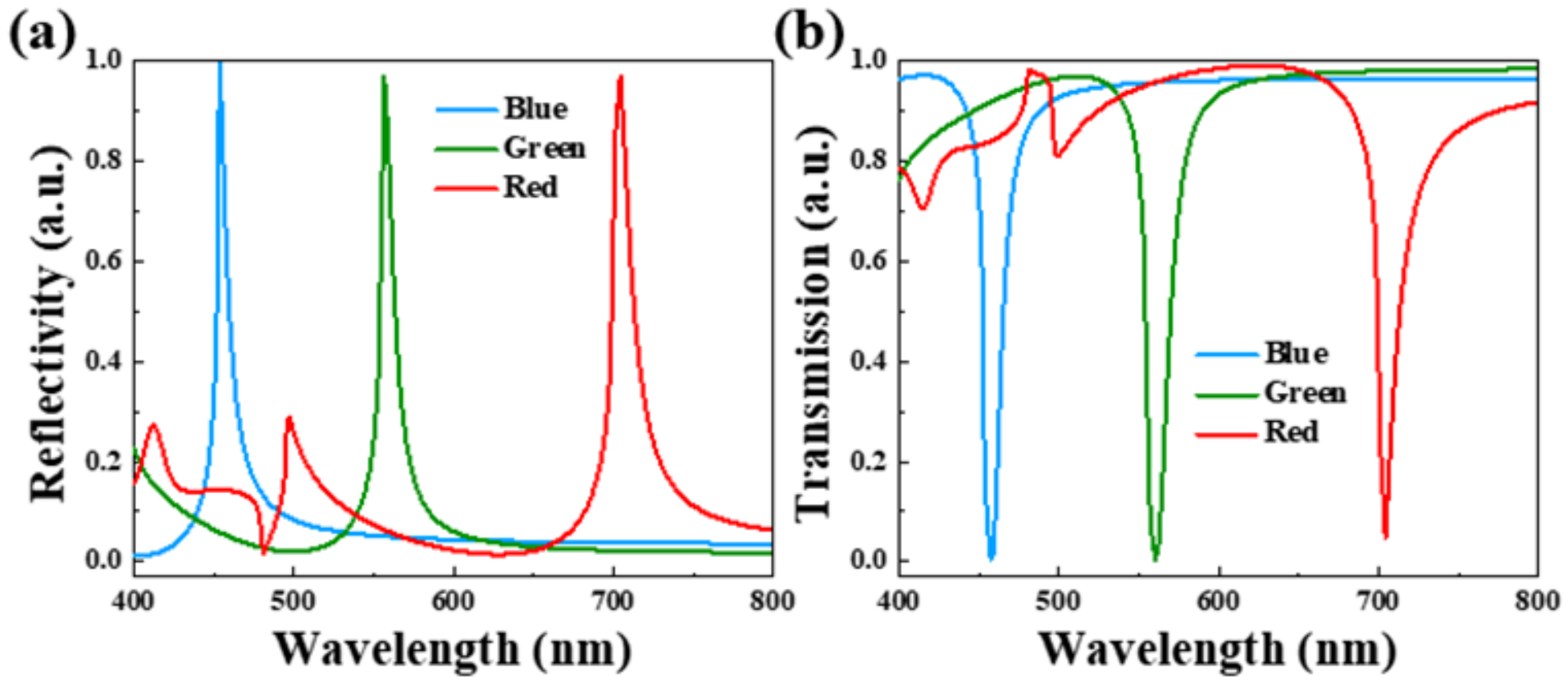

Figure 2

(a) Reflection and (b) transmission RGB spectra of CF-1.

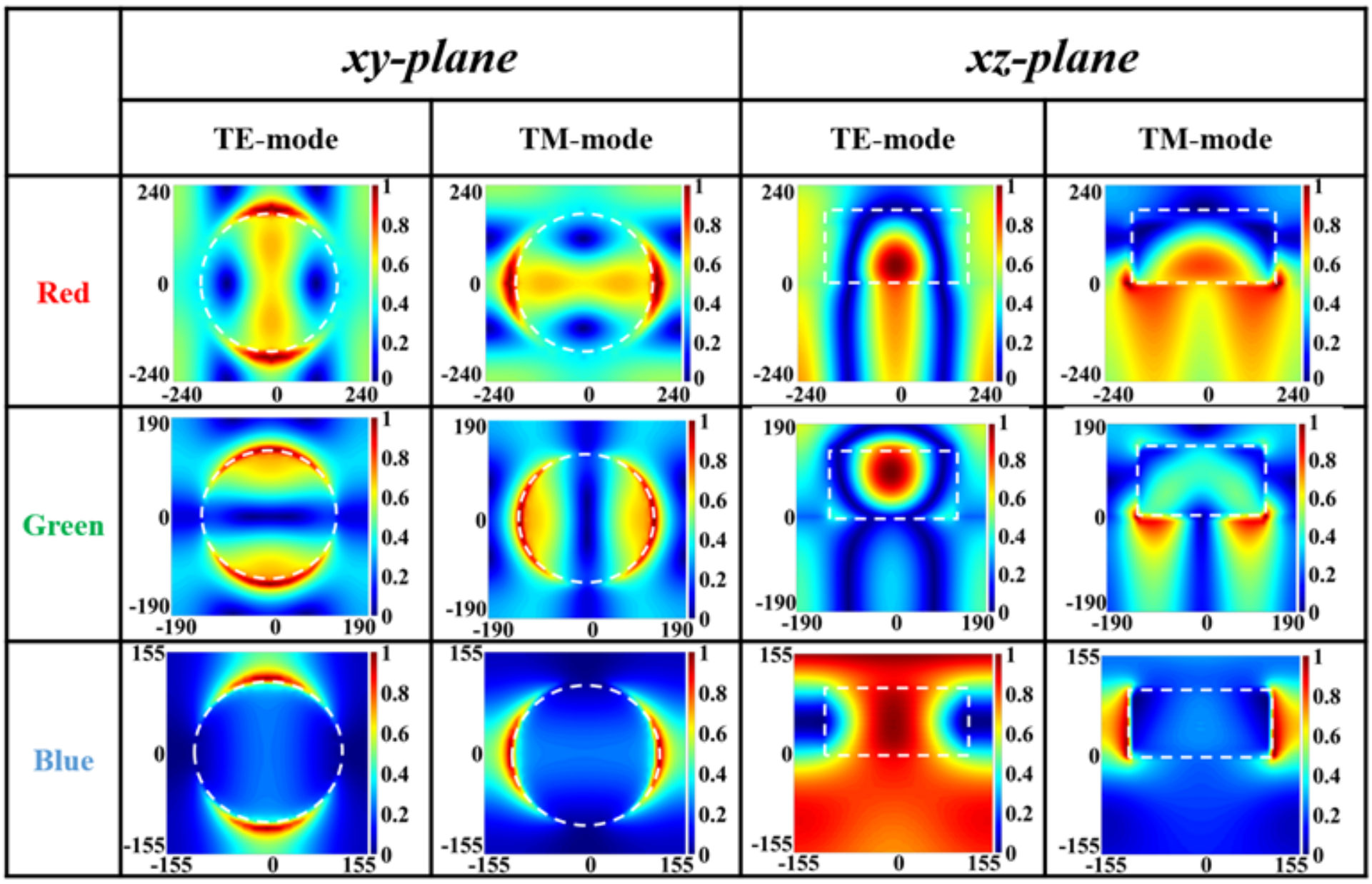

Figure 3 
E-field distributions on $x y$ - and $x z$-planes of RGB colors filtering characteristics in TE and TM modes for CF-1.
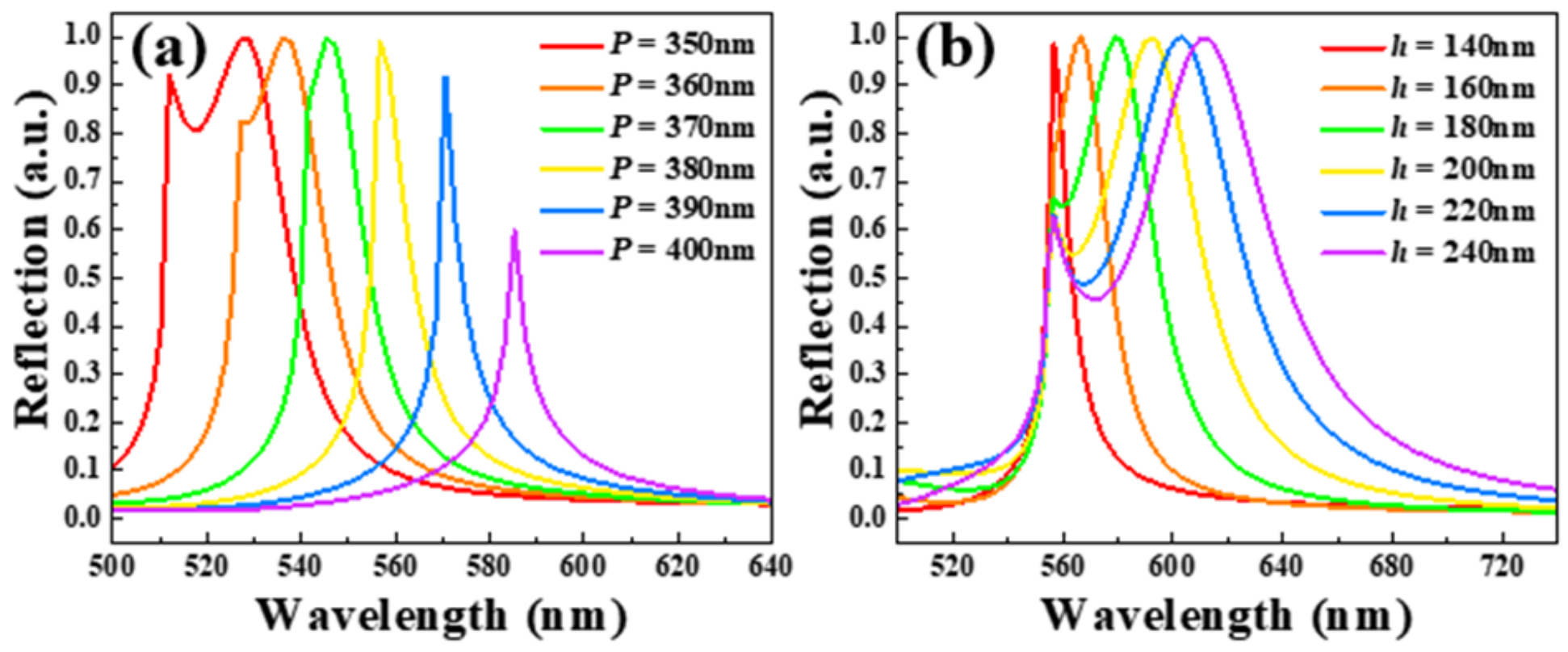

Figure 4

Reflection spectra of CF-1 with different (a) P and (b) h values for green color filtering characteristic.
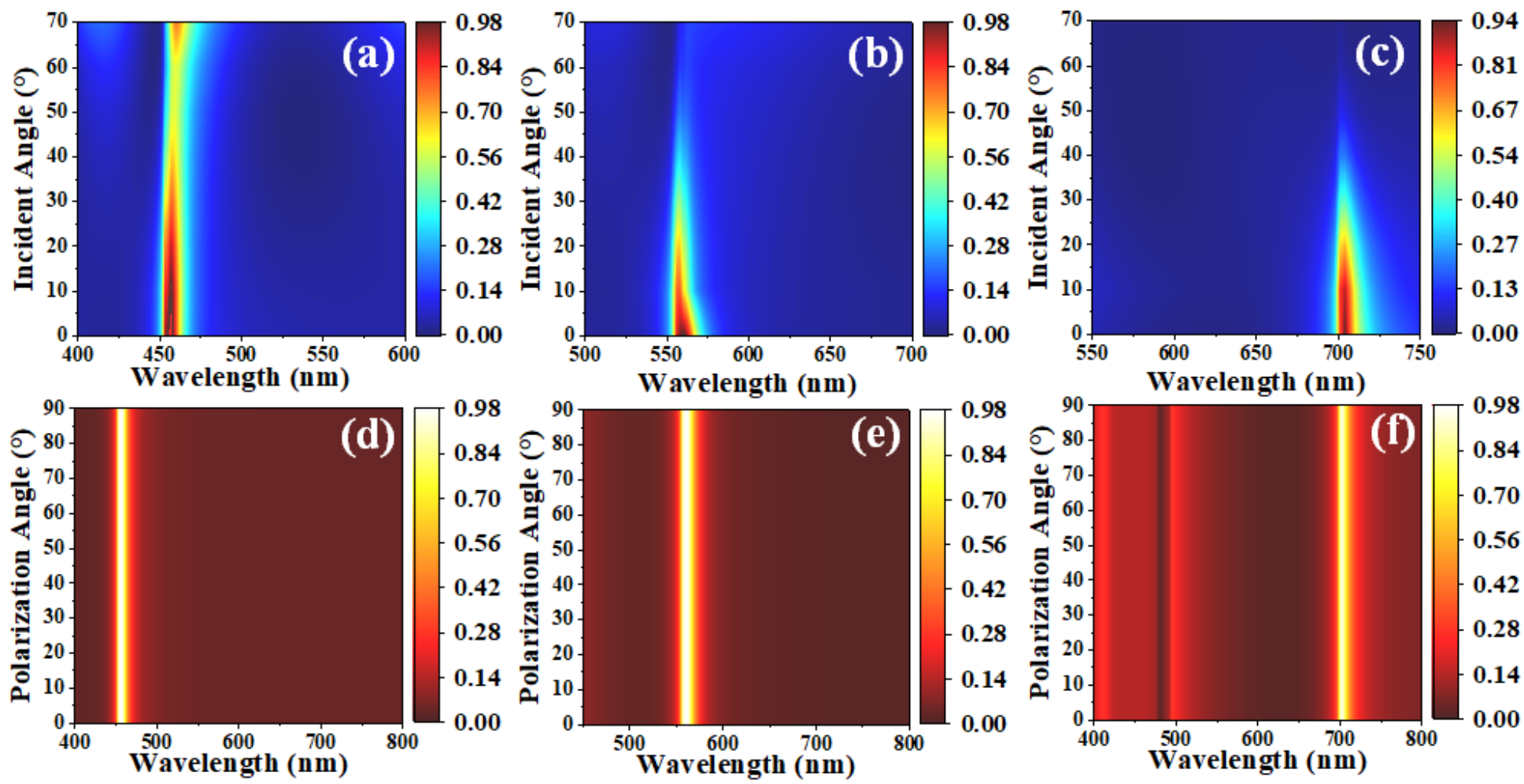

Figure 5

The contour maps of reflection spectra of CF-1 with different (a-c) incident and (d-f) polarization angles for (a)(d) blue, (b) (e) green, and (c)(f) red colors filtering characteristics. 
(a)

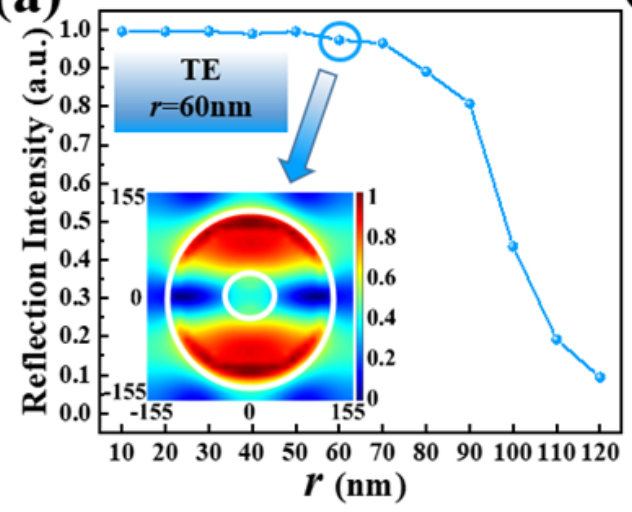

(b)

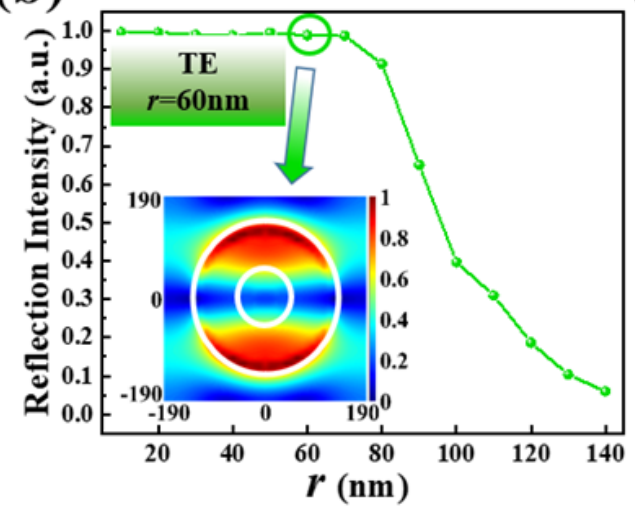

(c)

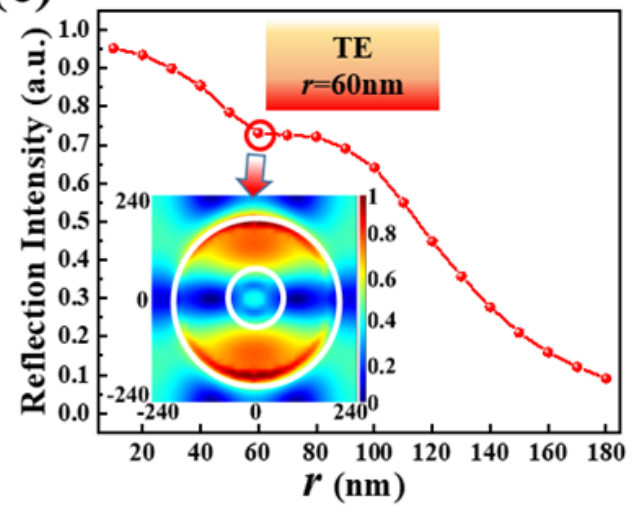

Figure 6

Reflection intensities of CF-2 with different $r$ values for (a) blue, (b) green, and (c) red colors filtering characteristics. The inserted images are the E-field distributions of CF-2 with $r=60 \mathrm{~nm}$ in TE mode.
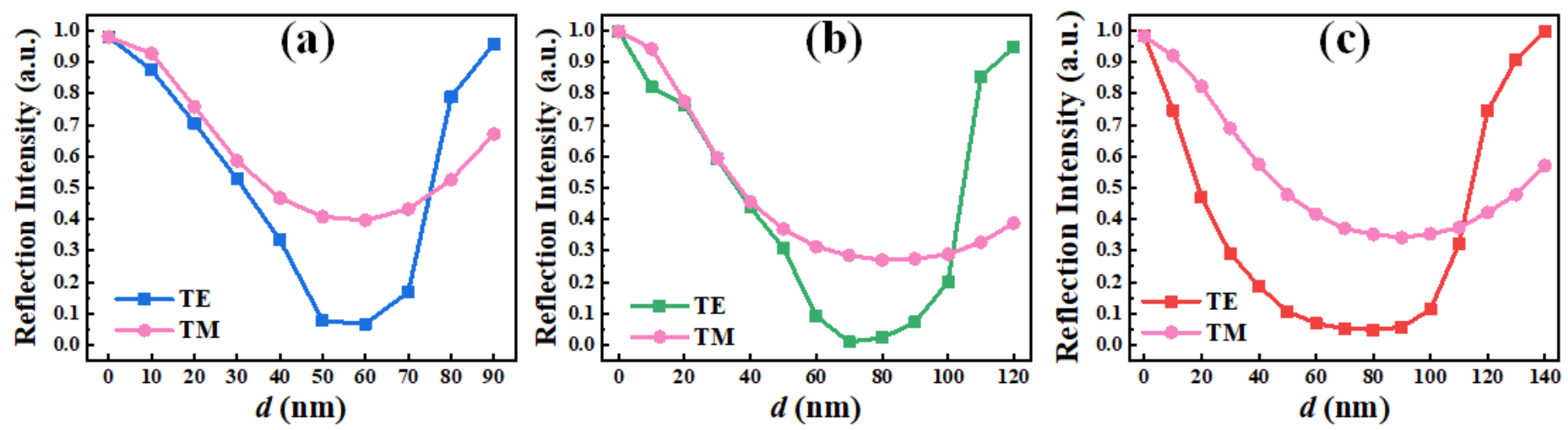

Figure 7

Reflection intensities of CF-3 with different $d$ values for (a) blue, (b) green, and (c) red colors filtering characteristics in TE and TM modes. 

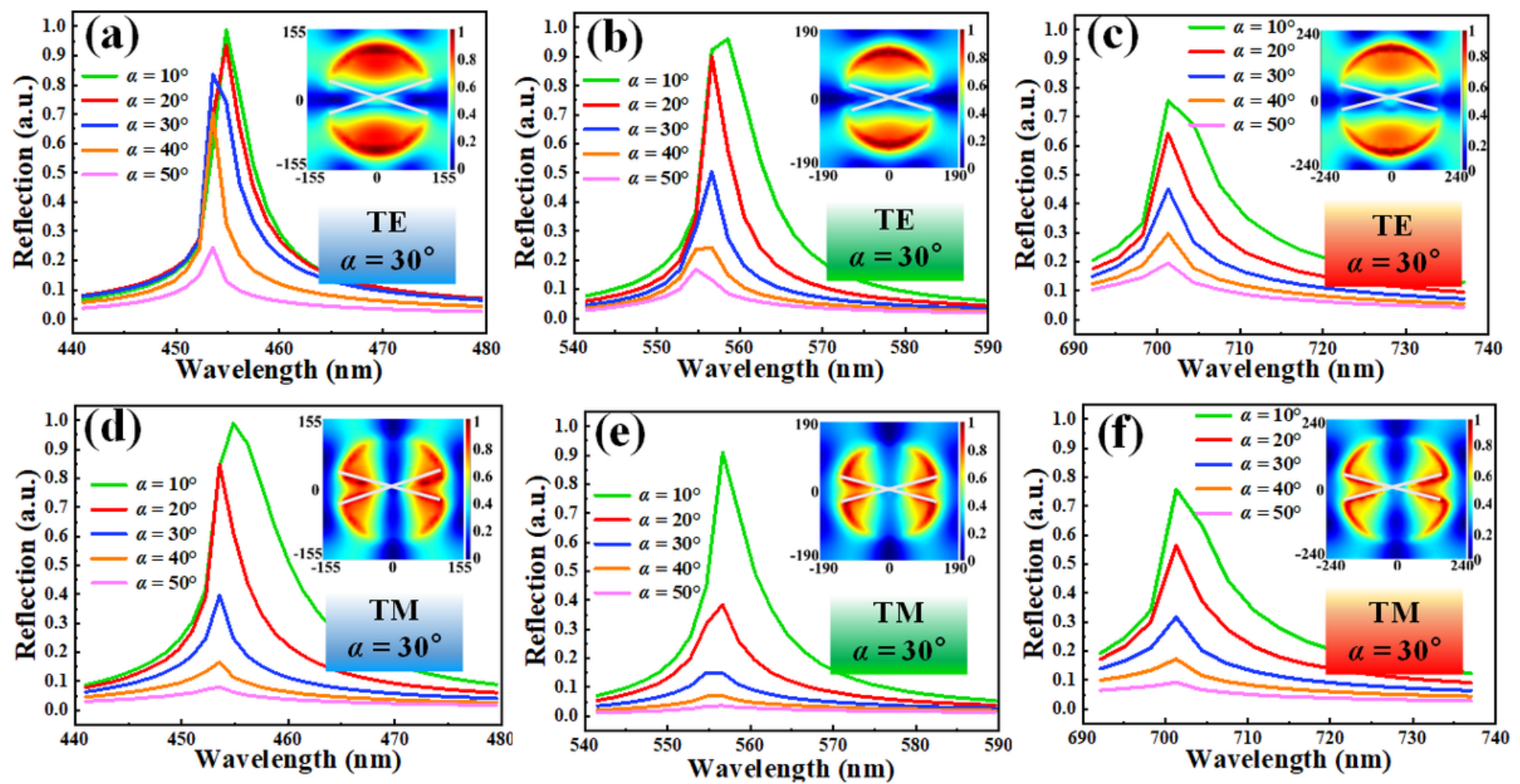

Figure 8

Reflection spectra of CF-4 with different a values in (a-c) TE and (d-f) TM modes for (a)(d) blue, (b)(e) green, and (c)(f) red colors filtering characteristics. The inserted images are E-field distributions of CF-4 with $a=30^{\circ}$. 